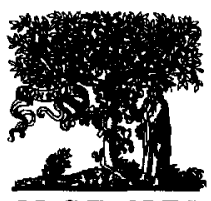

ELSEVIER

J. Non-Newtonian Fluid Mech., 60 (1995) $199-223$



\title{
Efficient simulation of nonlinear viscoelastic fluid flows
}

\author{
P. Saramito \\ Laboratoire de Rhéologie, BP53, 3804I, Grenohle cedex, France
}

Received 22 March 1993: in revised form 13 June 1995

\begin{abstract}
This paper presents a new and efficient method for computing the flow of a non-Newtonian fluid. The approach is based on two independent concepts:

Time-dependent simulation of viscoelastic flow: A new decoupled algorithm, presented in P. Saramito, Simulation numérique d'ecoulements de fluides viscoélastiques par éléments finis incompressibles et une méthode de directions alternées; applications, Thèse de l'Institut National Polytechnique de Grenoble, 1990 and P. Saramito, Numerical simulation of viscoelastic fluid flows using incompressible finite element method and a $\theta$-method, Math. Modelling Num. Anal., 35 (1994) 1-35. enables us to split the major difficulties of this problem, and to solve it more efficiently. Moreover, this scheme is of order two in time. and can be used to obtain stationary flows in an efficient way.

Conservative finite element method: this method combines the incompressible Raviart Thomas element, the discontinuous Lesaint-Raviart element. and a finite volume element method. It satisfies exactly the mass conservation law, and leads to an optimal size for the nonlinear system in terms of the total degree of freedom versus the mesh size.

We apply our numerical procedure to the Phan-Thien-Tanner model with a classical benchmark: the four to one abrupt contraction. The numerical solutions exhibit good behavior. especially near the singularity, in the vicinity of the re-entrant corner. The numerical experiments present the main features of such flows: vortex development and overshooting of the velocity profile along the axis of symmetry in the entry region.
\end{abstract}

Kevwords: Viscoelastic fluids: Viscoelastic flow

\section{Introduction}

The numerical simulation of viscoelastic fluid flows is a major challenge: it appears as both a test for assessing the robustness and efficiency of numerical methods, and a qualification of models for complex flow geometries. 
Intensive research has pointed out the theoretical difficulties in solving the large nonlinear system obtained from the approximation of the boundary value problem. Keunings [23] provides an exhaustive survey of the considerable work performed in this domain. There are three main difficulties in the previous approaches:

- The so-called high Weissenberg number problem: simulations were stopping before a limit value (approximately 4) of this dimensionless parameter, which expresses the memory effect of the material. It is now well accepted that the reasons for this difficulty are numerical: the use of inappropriate boundary conditions and numerical schemes associated with the type of governing equations [22].

- The approximation strategy: finite element spaces have to satisfy compatibility conditions (i.e. the well-known inf-sup conditions [2,5,13]). These conditions guarantee that the approximate problem is well-posed and that convergence occurs with mesh refinement.

- The tremendous amount of computation needed, thus limiting the size of the system that can be simulated.

In 1987, Crochet and Marchal [7] presented the first result above the "critical" Weissenberg number. They solved the stationary problem by using Newton's algorithm and a global Gaussian inversion of the Jacobian matrix. This direct method allowed them to reach numerical results using the Oldroyd model for Weissenberg numbers $(W e$ ) up to 14 for a plane contraction, and $W e=20.8$ in the axisymmetric case, using two meshes ( $W e=64$ for the lower mesh). The authors presented a subelement approximation strategy for stress tensor components. In this approach the number of degrees of freedom of the system was large, therefore requiring considerable computing time on a supercomputer.

In 1989, Fortin and Fortin [11] proposed a decoupled algorithm, based on the GMRES method, for solving the stationary problem of an Oldroyd fluid. They developed discontinuous approximation of the stress tensor. Using this approach, they obtained up to $W e=8$, with the Oldroyd model and for a plane contraction. However, their solutions present oscillations: the authors pointed out that stress schemes were not strictly TVD (total variation diminishing) and that computing time was important on a workstation.

We propose here an efficient method for computing flows of non-Newtonian fluids. Moreover, we apply this method by using a realistic viscoelastic model: the Phan-Thien-Tanner model. Section 2 develops constitutive and conservation laws of viscoelastic fluids of the Phan-Thien-Tanner type. Section 3 presents the study of viscometric properties and one-dimensional Poiseuille fluid flow, using PhanThien-Tanner model.

In Sections 4 and 5 we develop our numerical approach. On the one hand, we use a time-dependent decoupled algorithm [36,35]. As shown in Section 4, this algorithm enables us to split the major difficulties of this problem, and to solve it more efficiently. Moreover, this scheme is of order two in time, and can be used to obtain stationary flows in an efficient way. On the other hand, we develop an original conservative finite element approximation of the problem in Section 5. This element is based on a combination of the incompressible Raviart-Thomas element, the discontinuous Lesaint-Raviart element, and a finite volume element method. It 
satisfies exactly the mass conservation law, and leads to an optimal size for the nonlinear system, in terms of the total degree of freedom versus mesh size.

We apply our numerical procedure to the Phan-Thien-Tanner model with a classical benchmark: the four to one abrupt contraction (Sections 6 and 7). By drawing a first normal extra-stress component near the re-entrant corner, we can check the high quality of the numerical approximation. Then, we point out vortex development, and overshooting of the velocity profile along the axis of symmetry in the entry region.

\section{The governing equations}

In this section we present the formulation of constitutive and conservative laws, and the mathematical formulation of the problem.

\subsection{Constitutive law's}

The Cauchy stress tensor is given by the relation:

$$
\sigma=-p I+2 \eta_{n} D(\boldsymbol{u})+\tau
$$

We use the following notations: $p$ is the pressure field, $\boldsymbol{u}$ is the velocity field, $\tau$ is an extra-stress tensor. In addition, $D(\boldsymbol{u})=\left(\nabla \boldsymbol{u}+\nabla \boldsymbol{u}^{\prime}\right) / 2$ denotes the rate-of-deformation tensor, and $\eta_{n}$ represents the Newtonian viscosity of the fluid. The extra-stress t satisfies a constitutive law. First, we consider the Oldroyd model [30],

$$
\lambda \tau+\tau-2 \eta_{i} D(\boldsymbol{u})=0,
$$

where $i$ is the relaxation time of the fluid memory, $\eta_{1}$ a second viscosity, and $($. represents a material derivation of a tensor $[30,26]$

$$
\tau=\frac{\partial \tau}{\partial t}+\boldsymbol{u} \cdot \nabla \tau-W(\boldsymbol{u}) \cdot \tau+\tau \cdot W(\boldsymbol{u})-a(D(\boldsymbol{u}) \cdot \tau+\tau \cdot D(\boldsymbol{u})) .
$$

In the last relation, $a$ is related to a material parameter: $a \in[-1,1]$. and $W(\boldsymbol{u})=\left(\nabla \boldsymbol{u}-\nabla \boldsymbol{u}^{t}\right) / 2$ is the vorticity tensor.

This model includes a retardation time $\mu=\alpha \lambda_{n}$, where $x=\eta_{1} /\left(\eta_{n}+\eta_{1}\right)$ is a retardation parameter. Let us denote by $\eta=\eta_{n}+\eta_{\text {r }}$ the total viscosity. For $|a|<1$ in (2), we obtain the Johnson-Segalman model, and for $\eta_{n}=0$, the Maxwell model.

Some extra properties can be obtained by modifying the Oldroyd model (2): the Phan-Thien-Tanner model $[38,27]$ is given by the following constitutive equation:

$$
\lambda \tau+\exp \left(\frac{\epsilon \dot{\lambda}}{\eta_{r}} \operatorname{tr}(\tau)\right)-2 \eta_{v} D(u)=0
$$

where $\operatorname{tr}(\cdot)$ is the trace of a tensor, and $\epsilon$ is a parameter of the model. This gives rise to the following properties:

- the shear viscosity $\eta_{s}(\dot{\gamma})$ decreases with the shear rate $;$ (for $|a|<1$ ), and

- the elongational viscosity $\eta^{\prime}(\dot{\epsilon})$ reaches a maximum (for $\epsilon>0$ ). 


\subsection{Equations of conservation}

The momentum conservation law leads to

$$
\rho\left(\frac{\partial \boldsymbol{u}}{\partial t}+\boldsymbol{u} \cdot \nabla \boldsymbol{u}\right)+\nabla p-\eta_{\mathrm{n}} \Delta \boldsymbol{u}-\operatorname{div} \tau=0
$$

where $\rho$ is the (constant) density of the fluid. Mass conservation leads to the incompressibility relation:

$$
\operatorname{div} \boldsymbol{u}=0 .
$$

\subsection{Dimensionless parameters}

Let $U$ and $L$ be a typical velocity and length of the flow. We introduce the Weissenberg ${ }^{1}$ number $W e=\lambda U / L$, the Reynolds number $R e=\rho U L / \eta$, a retardation parameter [21] $\alpha=\eta_{\mathrm{v}} /\left(\eta_{\mathrm{n}}+\eta_{\mathrm{v}}\right)$, and we set

$$
x=\frac{\tilde{x}}{L}, \quad \boldsymbol{u}=\frac{\tilde{\boldsymbol{u}}}{U}, \quad t=\frac{U}{L} \tilde{t}, \quad \tau=\frac{L}{\eta U} \tilde{\tau}, \quad p=\frac{\tilde{p}}{\rho U^{2}} .
$$

where tildes are attached to dimensional variables. The dimensionless constitutive momentum equations are

$$
\begin{array}{ll}
\frac{W e}{2 \alpha} \vec{\tau}+\frac{f(\tau)}{2 \alpha} \tau-D(\boldsymbol{u}) & =0, \\
-\operatorname{Re}\left(\frac{\partial \boldsymbol{u}}{\partial t}+\boldsymbol{u} \cdot \nabla \boldsymbol{u}\right)+\operatorname{div} \tau+(1-\alpha) \Delta \boldsymbol{u}-\nabla p & =0, \\
\operatorname{div} \boldsymbol{u} & =0,
\end{array}
$$

where

$$
f(\tau)=\exp \left(\frac{\varepsilon W e}{\alpha} J \operatorname{tr} \tau\right)
$$

For $\varepsilon=0$, we obtain a fluid of Oldroyd type.

\subsection{Mathematical formulation}

The set of equations (8)-(10) have to be used together with initial and boundary conditions. For $\alpha \in[0,1[$ and $a=1$, the system (8)-(10) is found to be of mixed parabolic-hyperbolic type [22,33]. Let $\Omega$ be the flow domain. The required boundary and initial conditions are as follows:

-a velocity condition on the boundary $\Gamma=\partial \Omega$ :

$$
\boldsymbol{u}=\boldsymbol{u}_{\Gamma} \text { on } \Gamma \text {. }
$$

-a stress condition on the inflow boundary section: $\Gamma_{-}=\{x \in \Gamma ;(\boldsymbol{u} \cdot \boldsymbol{n})(x)<0\}$ :

$$
\tau=\tau_{\Gamma} \text { on } \Gamma_{-},
$$

\footnotetext{
' This formal definition will be applied in Section 3.4 to the Poiseuille flow, and flows in contraction.
} 
where $\boldsymbol{n}$ is the outward unit normal vector to $\Omega$ at the boundary $\Gamma$; -initial conditions for $\tau$ and $\boldsymbol{u}$ :

$$
\tau_{(t=1)}=\tau_{0}, \quad \boldsymbol{u}_{(t=0)}=\boldsymbol{u}_{0} \text { in } \Omega \text {. }
$$

Some results concerning the existence of solutions $(\tau, \boldsymbol{u}, p)$ of Eqs. (8)-(10), (12) (14) are known. Renardy [32] has obtained existence of stationary solutions for any value of $\alpha, 0 \leq x \leq 1$. Guillopé and Saut [20] have proved a global existence result of the solution in the unsteady case, for small values of $\alpha$.

The existence results are obtained for a fluid of Oldroyd type, and are valid under assumptions made for boundary and initial data $\boldsymbol{u}_{\mathrm{I}^{-}}, \tau_{\Gamma}, \boldsymbol{u}_{0}, \tau_{0}$.

\section{Steady simple flows}

This section briefly presents the viscometric data of Phan-Thien-Tanner fluids, and the choice of material parameters. We also study here steady Poiseuille flows. We use Poiseuille flows for the computation of inflow and outflow boundary conditions in an abrupt contraction (Section 7).

Finally. we precise the formal definition of the Weissenberg number of Section 2 , and give a practical one for converging flows: this definition takes into account elongational properties of Phan-Thien-Tanner fluids. Moreover, Section 7 shows that this practical definition fits vortex activity in an abrupt contraction.

\subsection{Elongational flows}

The version of the Phan-Thien-Tanner model with an exponential relaxation coefficient (4) is chosen here, because the exponential term results in a maximum in the steady state elongational viscosity. This behaviour (Fig. 1a) is in better
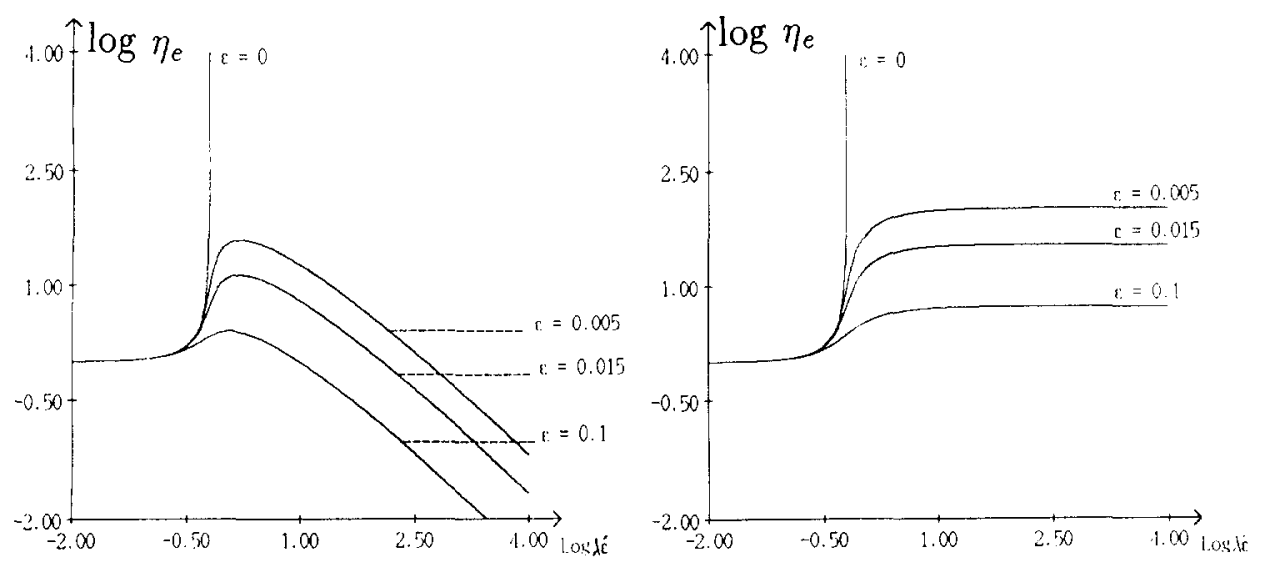

Fig. 1. Normalized steady state elongational properties of Phan-Thien-Tanner model, $a=0.8$. $x=0.875$ : (a) exponential law, (b) linear law. 

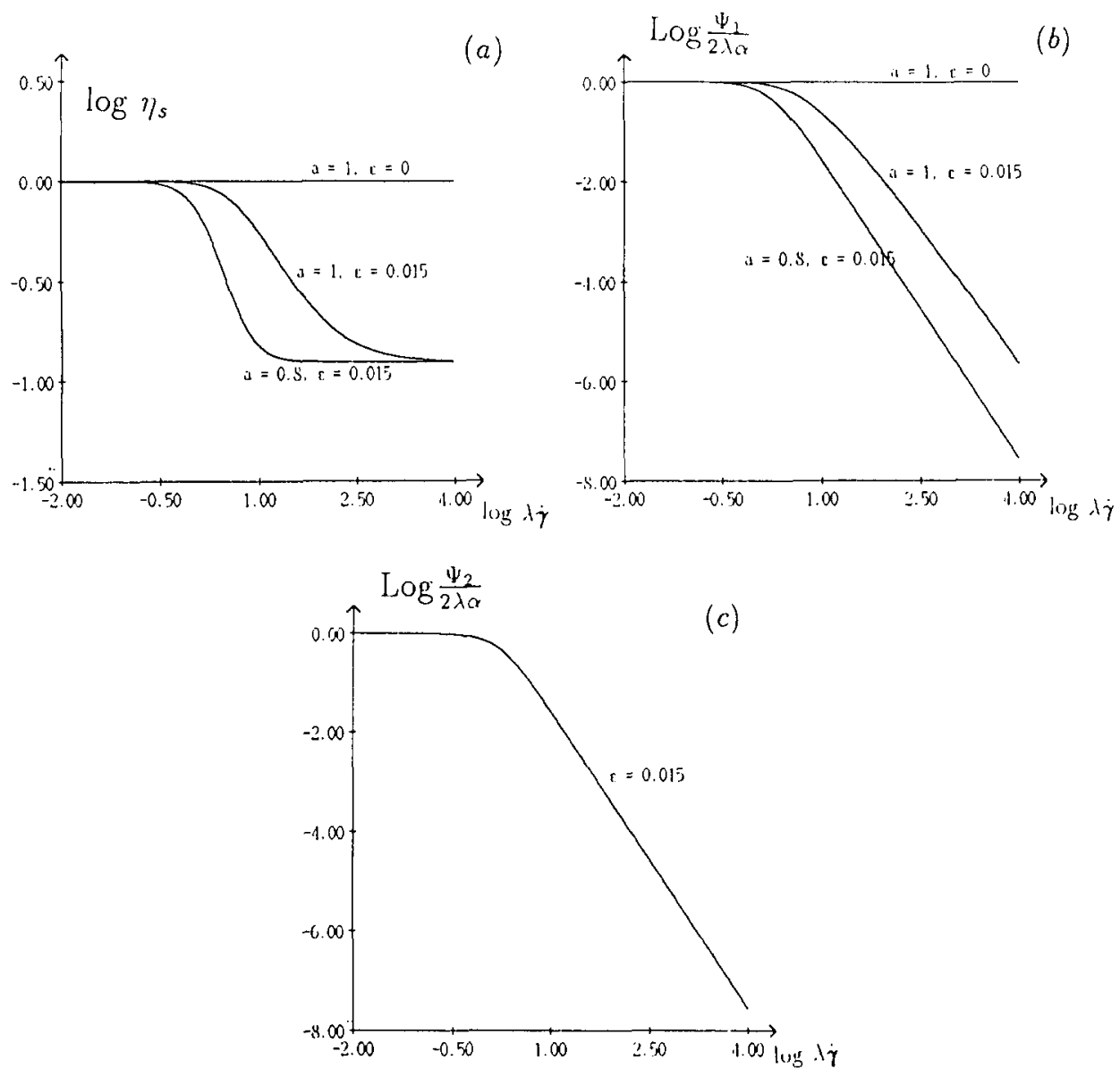

Fig. 2. Phan-Thien-Tanner model $(x=0.875)$ : Normalized shear viscosity (a), first normal stress difference (b), and second normal stress difference (c).

agreement with experimental data for polymer melts [26, p. 204] than the version with a linear coefficient (Fig. 1b). Note that the Johnson-Segalman model $(\varepsilon=0)$ predicts an infinite value for the steady state elongational viscosity.

\subsection{Simple shear flows}

The Phan-Thien-Tanner model shows shear thinning and a non-constant first normal stress coefficient (Fig. 2a). Let $f_{\mathrm{s}}(\hat{\lambda} \dot{\gamma})$ be the unique value satisfying

$$
f_{\mathrm{s}}(\lambda \dot{\gamma})=\exp \left(\frac{2 \varepsilon a(\lambda \dot{\gamma})^{2}}{f_{\mathrm{s}}(\lambda \dot{\gamma})^{2}+\left(1-a^{2}\right)(\lambda \dot{\gamma})^{2}}\right) .
$$

Then, the shear viscosity is given by 


$$
\eta_{\mathrm{s}} \stackrel{\text { def }}{=} \frac{\sigma_{12}}{\eta \dot{\gamma}}=1-\alpha+\alpha \frac{f_{\mathrm{s}}(\hat{\lambda} \dot{\gamma})}{f_{\mathrm{s}}(\hat{\lambda} \dot{\gamma})^{2}+\left(1-a^{2}\right)(\dot{\lambda} \dot{i})^{2}} .
$$

The model predicts first and second normalized stress difference, $\psi_{1}$ and $\psi_{2}$, respectively (see also Figs. $2 \mathrm{~b}, \mathrm{c}$ ):

$$
\frac{\psi_{1}}{2 \alpha \lambda} \stackrel{\text { def }}{=} \frac{\sigma_{11}-\sigma_{22}}{2 \alpha \lambda \dot{\dot{j}^{2}}}=\frac{1}{f_{\mathrm{s}}(\hat{\lambda} \dot{\gamma})^{2}+\left(1-a^{2}\right)(\hat{\lambda} \dot{\gamma})^{2}},
$$

and $\psi_{2} \stackrel{\text { def }}{=}\left(\sigma_{22}-\sigma_{33}\right) / \dot{\gamma}^{2}=(1-a) \psi_{1}$. Therefore, the second normal stress difference vanishes for $a=1$ (upper-convected derivative).

Nevertheless, for $|a|<1$ and up to a critical value $\alpha_{c}(\varepsilon)$ of $\alpha$, the model predicts an excessive shear thinning; in this case, the shear stress $\sigma_{12}=\eta_{\mathrm{s}} \dot{\gamma}$ becomes non-monotonic (Fig. 3). Conversely, when $0 \leq \alpha \leq \alpha_{c}(\varepsilon)$, the shear stress is always monotonic. For the Johnson-Segalman model, we have $\alpha_{c}(0)=8 / 9$.

\subsection{Poisenille flows}

We find in Refs. [21,29] an analytical resolution and stability study for Poiseuille flows of Oldroyd fluids. We need here a numerical approach to solve the Poiseuille flow of a Phan-Thien-Tanner fluid. Both analytical and numerical approaches start by reducing the one-dimensional Poiseuille problem to a scalar nonlinear characteristic equation. We solve the characteristic equation and obtain numerical solutions by a continuation algorithm.

Such solutions can exhibit pathological cases depending on material parameter values: we point out loss of existence or unicity of solutions, and existence of non-regular solutions.

Let $g$ and $2 g$ be the constant pressure gradients driving the plane and axisymmetric Poiseuille flows, respectively. By integration of the momentum conservation law, we obtain the characteristic equation

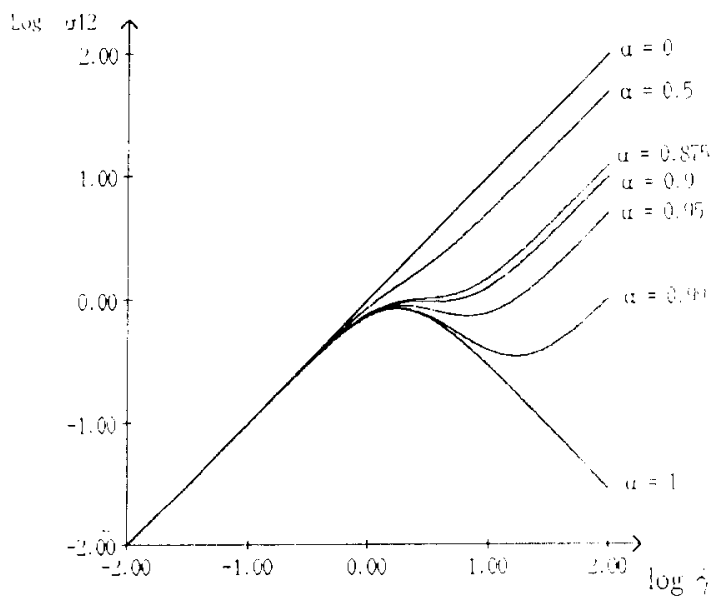

Fig. 3. Shear stress as predicted by the Phan-Thien- Tanner model: $\lambda=1, a=0.8$ and $\imath=0.015$ 


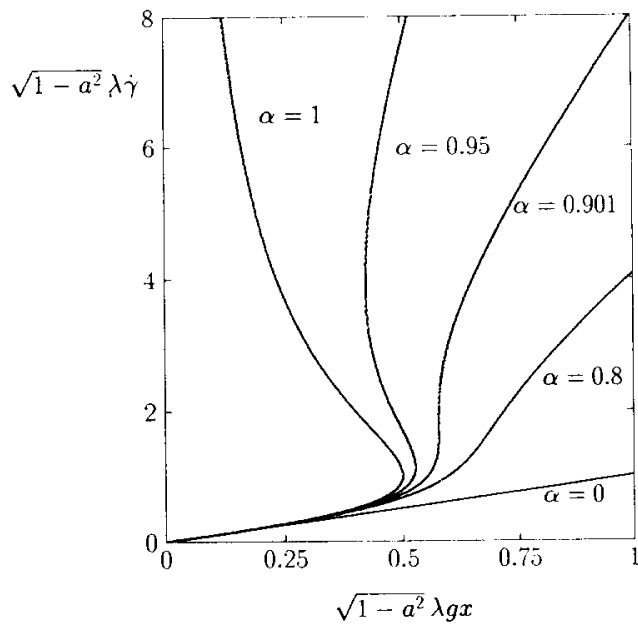

Fig. 4. Characteristic curves for the Phan-Thien-Tanner model: $\varepsilon=0.015$

$$
g x=\eta_{\mathrm{s}} \dot{\gamma}, \quad 0 \leq x \leq r,
$$

where $r$ is the radius of the section. Fig. 4 shows the characteristic curves of the fluid: $\sqrt{1-a^{2}} \lambda \dot{\gamma}$ versus $\sqrt{1-a^{2}} \lambda g x$. Up to the critical value $\alpha_{c}(\varepsilon)$ of $\alpha$, the characteristic curve admits two turning-back points denoted by $\chi^{-}$and $\chi^{+}$, $0 \leq \chi^{-} \leq \chi^{+}$. The situation is characterized as follows:

-For $0<\alpha<\alpha_{\mathrm{c}}(\varepsilon)$, we obtain one regular solution (Figs. 5a-c).

-For $\alpha=\alpha_{\mathrm{c}}(\varepsilon), \chi^{+}=\chi^{-}$, this solution remains regular if $\sqrt{1-a^{2}} \lambda g r \leq \chi^{-}$(Fig. $5 \mathrm{~d}$ ), and becomes singular otherwise (Figs. 5e,f).

-For $\alpha_{c}(\varepsilon)<\alpha<1$, we have one regular solution if $\sqrt{1-a^{2}} \lambda g r \leq \chi^{-}$(Fig. $5 \mathrm{~g}$ ), or a continuum of solutions if $\sqrt{1-a^{2}} \lambda g r>\chi^{-}$. Moreover, one of these solutions remains regular for $\sqrt{1-a^{2}} \lambda g r \leq \chi^{+}$(Fig. 5h). When $\sqrt{1-a^{2}} \lambda g r>$ $\chi^{+}$all solutions are singular (Fig. 5i), and present discontinuous rates of deformation.

-For $\alpha=1$ (Maxwell-like model), we have only one solution for $\sqrt{1-a^{2}} \lambda g r \leq$ $\chi^{+}$(Fig. $5 \mathrm{j}$ ), and no more solution otherwise.

There are different opinions in the literature about the interpretation of such solutions (i.e. when $\alpha>\alpha_{c}(\varepsilon)$ and high values of the rate of deformation appear near the wall). Malkus and co-workers [29] developed a description of a spurt flow as a trigger mechanism for the melt instabilities: they referred to this as an apparent slip, despite the fact that there is no actual slip, due to the relative thinness of the wall layer. However, as the pressure gradient increases, this wall layer thickens. Schowalter [9] proposes sharp velocity gradients near the wall as a possible model for fluid slip in the context of continuous velocity profile.

This model is not completely satisfactory: discontinuous rates of deformation have not been observed in the flow domain by experimenters, when macroscopic slip phenomena appear $[39,25,24]$. This leads us to argue that we have reached a possible limit of such differential models when a discontinuous rate of deformation appears. 
Nevertheless, this "rheological artifact" [27] is of great interest from mathematical and numerical points of view; it is helpful in understanding and writing constitutive equations. Moreover, the critical case before the appearance of a discontinuous rate of deformation can be viewed as a significant benchmark for testing the robustness and the efficiency of algorithms for viscoelastic flow problems. This last point is discussed in the following sections.

\subsection{Definition of the Weissenberg number}

We consider here the Poiseuille flow, which will be treated in the next paragraph. The shear-thinning character of the Phan-Thien-Tanner fluid makes it difficult to define $W e$ using directly $\dot{\gamma}$ at the wall. Instead, we use an average value $\overline{\dot{\gamma}_{w}}$ defined by ${\overline{\dot{\gamma}_{u}}}_{1}=4 \bar{U} / r$ for axisymmetric flow, and $\overline{\dot{\gamma}_{w}}=3 \bar{U} / r$ for plane flow where $\bar{U}$ denotes the average velocity. Let $W e$ be the Weissenberg number, as introduced in ref. [4] for the White-Metzner fluid:
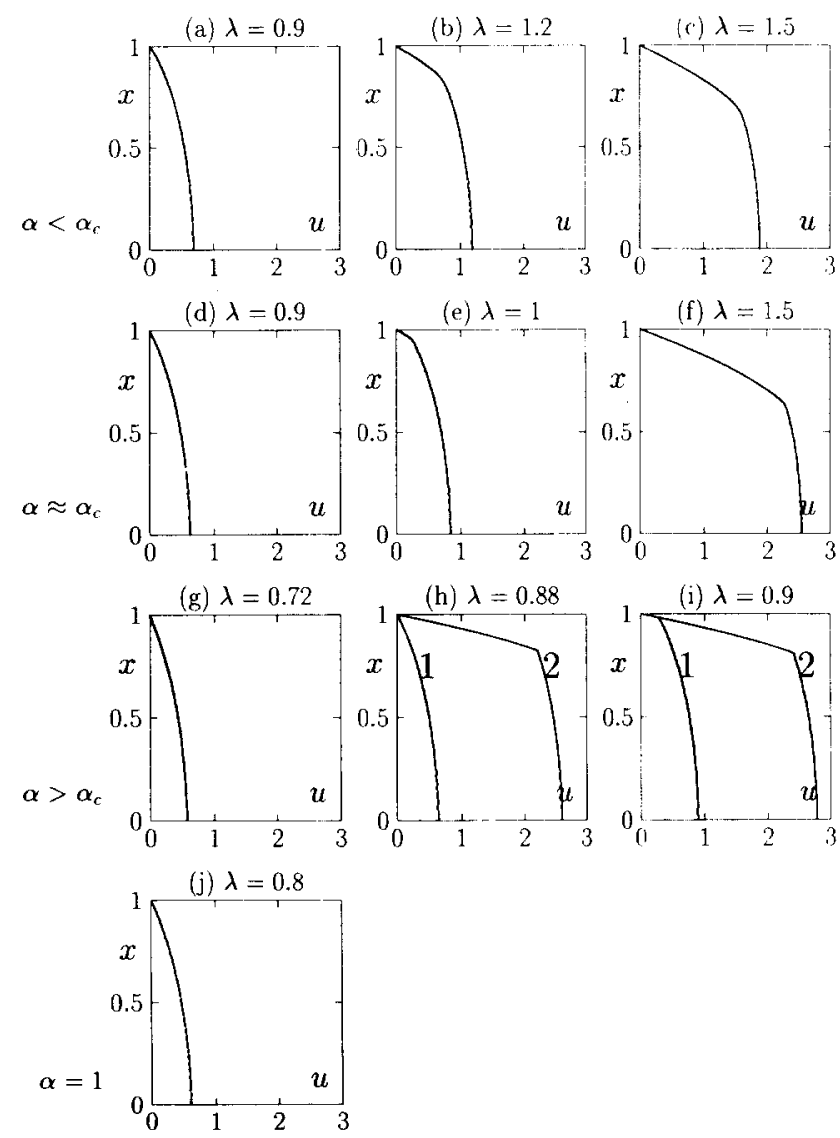

Fig. 5. Solutions of Poiseuille flow $(\varepsilon=0.015, g=1): x=0.875<x_{\mathrm{c}}: \lambda=0.9$ (a); $\lambda=1.2$ (b); $\lambda=1.5$ (c); $\alpha=0.901 \approx \alpha_{\mathrm{c}}: \lambda=0.9$ (d); $\lambda=1$ (c); $\lambda=1.5(\mathrm{f}) ; \alpha=0.95>\alpha_{\mathrm{c}}: \lambda=0.72(\mathrm{~g}) ; \lambda=0.8$ (h); $\lambda=0.9$ (i); $\alpha=1$ : $\lambda=0.8(\mathrm{j})$. 


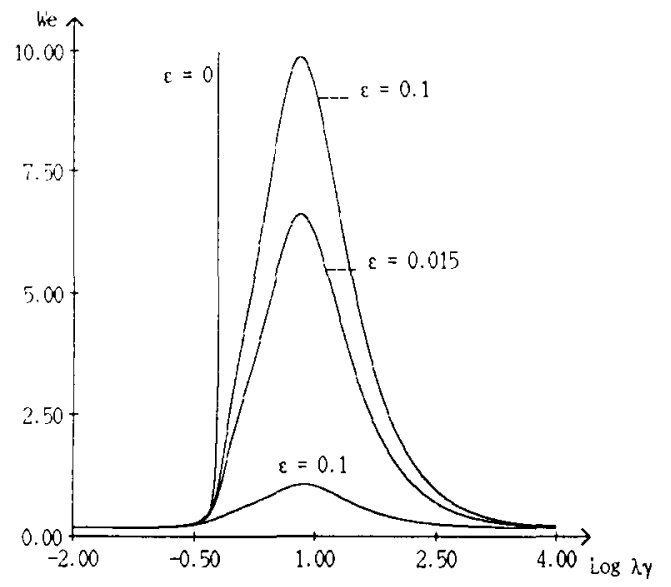

Fig. 6. Weissenberg number versus $\bar{\lambda} \overline{\dot{\gamma}_{w}} ; a=0.8, \varepsilon=0.015$.

$$
W e=\lambda_{\mathrm{n}} \overline{\dot{\gamma}_{\mathrm{w}}},
$$

where $\lambda_{\mathrm{n}}$ is the natural time of the fluid:

$$
\lambda_{\mathrm{n}}=\sigma_{E \mid \dot{s}=\overline{\dot{r}_{\mathrm{w}}}} / 6 \sigma_{12} \overline{\dot{\gamma}_{\mathrm{w}}} \text {. }
$$

Note that the definition (20) of the natural time $\lambda_{\mathrm{n}}$ uses the first normal stress difference $\sigma_{E}$ in uniaxial extension $\dot{\varepsilon}=\bar{\gamma}_{\mathrm{w}}$. A representative rate of elongation $\dot{\varepsilon}$ is given by: $\dot{\varepsilon}=\overline{\gamma_{w}}$. Thus the definition given in Ref. [4] for the White-Metzner fluid has been modified in order to include elongational properties. Moreover, Section 7 shows that this definition of We corresponds to vortex development.

Therefore, when $\varepsilon>0$, the Phan-Thien-Tanner model predicts bounded values of the Weissenberg number (Fig. 6). Conversely, with $\varepsilon=0$ (Johnson-Segalman and Oldroyd models), the Weissenberg number is not bounded for high values of $\lambda \overline{\dot{\gamma}_{w}}$. As pointed out in Ref. [4], the prediction of bounded values of $W e$ is in better agreement with experimental data.

Definition (19) of We applies to flow in contractions, by considering Poiseuille flow at the downstream section.

\section{Time approximation using a $\theta$-scheme}

We shall now restrict our study to the problem $(8)-(10),(12)-(14)$, where the inertia term $(\boldsymbol{u} \cdot \nabla) \boldsymbol{u}$ in (9) is neglected, since only slow flows are investigated.

In this section, we present a new method for solving unsteady viscoelastic problems. This method is related to the alternating direction implicit technique $[31,17,16]$ for decoupling the main two difficulties: the nonlinearities of Eq. (8), and the incompressibility relation (10). 


\subsection{Splitting the problem}

We consider the following two operators:

$$
\begin{aligned}
& A_{1}(\tau, \boldsymbol{u}, p)=\left[\begin{array}{c}
\frac{w}{2 \alpha} \tau-D(\boldsymbol{u}) \\
\operatorname{div} \tau+(1-\alpha) \Delta \boldsymbol{u}-\nabla p \\
\operatorname{div} \boldsymbol{u}
\end{array}\right], \\
& A_{2}(\tau, \boldsymbol{u}, p)=\left[\begin{array}{c}
\frac{W e}{2 \alpha} T(\boldsymbol{u}) \tau+\frac{f(\tau)-w}{2 \alpha} \tau \\
0 \\
0
\end{array}\right],
\end{aligned}
$$

As mentioned above, the advective part of the momentum equation $(\boldsymbol{u} \cdot \nabla) \boldsymbol{u}$ is neglected, since only slow flows are investigated.

In (21)-(22), we introduce $w \in] 0,1$, a parameter associated with the splitting. The Laplace operator $\Delta$ in (21) is related to the boundary conditions (12) for $\boldsymbol{u}$, and the transport operator $T(\boldsymbol{u}) \stackrel{\text { def }}{=}(\boldsymbol{u} \cdot \nabla)+\beta_{a}(\cdot, \nabla \boldsymbol{u})$ in $(22)$ is related to the inflow boundary condition (13) for $\tau$. The bilinear form $\beta_{a r}(\cdot, \cdot)$ is defined by

$$
\beta_{a}(\tau, \nabla \boldsymbol{u})=\tau \cdot W(\boldsymbol{u})-W(\boldsymbol{u}) \cdot \tau-a(D(\boldsymbol{u}) \cdot \tau+\tau \cdot D(\boldsymbol{u})) .
$$

Let us consider the following problem:

find $\mathscr{U}=(\tau, \boldsymbol{u}, p)$ defined in $\Omega$, such that

$$
m \frac{\mathrm{d} U}{\mathrm{~d} t}+A_{1}(\mathscr{U})+A_{2}(\mathscr{U})=0
$$

and satisfying the initial condition (14). The diagonal matrix in (24) is defined by $m=\operatorname{diag}(W e / 2 \alpha,-R e, 0)$. The parameter $w$ is related to a numerical relaxation parameter of the splitting.

\subsection{Time approximation}

Using these definitions, we build a time approximation sequence $\mathscr{U}^{n}=\left(\tau^{n}, \boldsymbol{u}^{\prime \prime}, p^{\prime \prime}\right)$ of a solution $\mathscr{U}(t)=(\tau(t), u(t), p(t))$ of $(24)$. For a given $\mathscr{U}^{0}$, the sequence $\left(\mathscr{U}^{n}\right)_{n>0}$ is defined by the following three-step procedure [15]:

$$
\begin{aligned}
& m \frac{\mathscr{U}^{n+\theta}-\mathscr{U}^{n}}{\theta \Delta t}+A_{1}\left(\mathscr{U}^{n+\theta}\right)=-A_{2}\left(\mathscr{U}^{n}\right), \\
& m \frac{\mathscr{U}^{n+1-\theta}-\mathscr{U}^{n+\theta}}{(1-2 \theta) \Delta t}+A_{2}\left(\mathscr{U}^{n+1-\theta}\right)=-A_{1}\left(\mathscr{U}^{n+\theta}\right) . \\
& m \frac{\mathscr{U}^{n+1}-\mathscr{U}^{n+1-\theta}}{\theta \Delta t}+A_{1}\left(\mathscr{U}^{n+1}\right)=-A_{2}\left(\mathscr{U}^{n+1-\theta}\right) .
\end{aligned}
$$

where $\Delta t$ is the total time step, and $\theta \in] 0, \frac{1}{2}[$ is the parameter of the time scheme. 


\subsection{The $\theta$-method}

The definitions of Section 4.1 and the time scheme of Section 4.2 lead to our new algorithm $[35,36]$ for solving unsteady flows of viscoelasticity fluids. This scheme is of order two in time, and allows us to compute steady solutions efficiently. We can also decouple the computation of stress and velocity-pressure; we get two standard sub-problems: Stokes-type, from steps (25) and (27), and transport type, from step (26).

First, let us study step 1. For $\tau^{n}, \boldsymbol{u}^{n}$ given, we search $\left(\tau^{n+\theta}, \boldsymbol{u}^{n+\theta}, p^{n+\theta}\right)$ such that

$$
\begin{aligned}
& \frac{W e}{2 \alpha} \frac{\tau^{n+\theta}-\tau^{n}}{\theta \Delta t}+\frac{w}{2 \alpha} \tau^{n+\theta}-D\left(\boldsymbol{u}^{n+\theta}\right)=-\frac{W e}{2 \alpha} T\left(\boldsymbol{u}^{n}\right) \tau^{n}-\frac{f\left(\tau^{n}\right)-w}{2 \alpha} \tau^{n}, \\
& -\operatorname{Re} \frac{\boldsymbol{u}^{n+\theta}-\boldsymbol{u}^{n}}{\theta \Delta t}+\operatorname{div} \tau^{n+\theta}+(1-\alpha) \Delta \boldsymbol{u}^{n+\theta}-\nabla p^{n+\theta}=0, \\
& \operatorname{div} \boldsymbol{u}^{n+\theta}=0, \\
& \boldsymbol{u}^{n+\theta}=\boldsymbol{u}_{\Gamma}((n+\theta) \Delta t) \text { on } \Gamma .
\end{aligned}
$$

We set

$$
\gamma^{n}=\left(W e-\left(f\left(\tau^{n}\right)-w\right) \theta \Delta t\right) \tau^{n}-(W e \theta \Delta t) T\left(\boldsymbol{u}^{n}\right) \tau^{n} .
$$

From (28), we get

$$
\tau^{n+\theta}=\frac{1}{W e+w \theta \Delta t}\left(\gamma^{n}+2 \theta \Delta t D\left(\boldsymbol{u}^{n+\theta}\right)\right) .
$$

Since $\operatorname{div} \boldsymbol{u}^{n+\theta}=0$, we have $2 \operatorname{div} D\left(\boldsymbol{u}^{n+\theta}\right)=\Delta \boldsymbol{u}^{n+\theta}$, and Eq. (29) becomes

$$
k \boldsymbol{u}^{n+\theta}-s \Delta \boldsymbol{u}^{n+\theta}=f^{n}
$$

with

$$
\begin{aligned}
& k=\operatorname{Re} /(\theta \Delta t), \\
& s=1-\alpha \frac{W e-(1-w) \theta \Delta t}{W e+w \theta \Delta t}
\end{aligned}
$$

and

$$
f^{n}=k \boldsymbol{u}^{n}+\frac{1}{W e+w \theta \Delta t} \operatorname{div} \gamma^{n} .
$$

Step 1 can be solved as follows:

$-\tau^{n}$ and $\boldsymbol{u}^{n}$ being known, compute successively $\gamma^{n}$ and $f^{n}$ using (32) and (37), respectively;

$-f^{n}$ being known, compute $\left(\boldsymbol{u}^{n+\theta}, p^{n+\theta}\right)$ as the solution of the Stokes-type problem (34) and (30) with the boundary condition (31);

$-\gamma^{n}$ and $\boldsymbol{u}^{n+\theta}$ being known, compute $\tau^{n+\theta}$ in an explicit way, using (33).

Now, we can study the second step. We search $\left(\tau^{n+1-\theta}, \boldsymbol{u}^{n+1-\theta}\right)$ such that 


$$
\begin{aligned}
& \frac{W e}{2 \alpha} \frac{\tau^{n+1-\theta}-\tau^{n+\theta}}{(1-2 \theta) \Delta t}+\frac{W e}{2 \alpha} T\left(\boldsymbol{u}^{n+1-\theta}\right) \tau^{n+1-\theta}+\frac{f\left(\tau^{n+1}+t^{\prime}\right)-w}{2 \alpha} \tau^{n+1 \ldots \prime \prime} \\
& \quad=-\frac{w}{2 \alpha} \tau^{n+\theta}+D\left(\boldsymbol{u}^{n+\theta}\right), \\
& -\operatorname{Re} \frac{\boldsymbol{u}^{n+1-\theta}-\boldsymbol{u}^{n+\theta}}{(1-2 \theta) \Delta t}=-\operatorname{div} \tau^{n+\theta}-(1-x) \Delta \boldsymbol{u}^{n+\theta}+\nabla p^{n+\theta}, \\
& \tau^{n+1-t^{\prime}}=\tau_{\Gamma}((n+1) \Delta t) \text { on } \Gamma .
\end{aligned}
$$

From (29) and (39), we obtain

$$
\boldsymbol{u}^{n+1-\theta}=\frac{1-\theta}{\theta} \boldsymbol{u}^{n+\theta}-\frac{1-2 \theta}{\theta} \boldsymbol{u}^{n} .
$$

Step 2 reduces to the transport-type problem (38) with the boundary condition (40). Moreover, using Oldroyd or Maxwell models $(f \equiv 1)$, this problem becomes linear in $\tau$. The resolution of step 3 can be deduced from (32)-(37) by substituting $n$, $(n+0)$ by $(n+1-\theta),(n+1)$, respectively. Finally, we can solve the time-dependent problem using a $\theta$-method. We obtain a succession of Stokes-type and transport-type problems.

The choice $\theta=1 / \sqrt{2}$ presents some good convergence properties, as shown in Ref. [37]. Since our numerical experiments on the Phan-Thien-Tanner model, and previously [35] on an Oldroyd-B model, are concerned, the parameter $w$ of the splitting has no influence on the convergence rate of the $\theta$-schema towards the stationary solution. The convergence rate is related to the residual term $A_{1}(\mathscr{U})+A_{2}(\mathscr{U})$ of the stationary problem. The time step $\Delta t$ is automatically adjusted during iterations, in order to optimize the convergence rate.

We point out that the $\theta$-method is independent of the approximation with respect to space: as a consequence, this method is worth using for other space-approximations than the one presented in the following section.

\subsection{Resolution of the sub-problems}

The sub-problems of Stokes-type are solved using a conjugate gradient method. The preconditioning is obtained by means of the augmented Lagrangian method [12]. The efficiency of this method is particularly spectacular for the test problem of Section 6, as the initial problem is ill-conditioned due to the domain length and the presence of the re-entrant corner.

The transport sub-problems are solved by using a symmetrized successive overrelaxation method (SSOR, see Ref. [18]). Numerical tests of this procedure have shown fast convergence of the residual terms.

\section{Approximation with respect to space}

In the finite element approach, the use of classical approximation methods for the velocity (for example, the $P_{2}-P_{1}$ element) leads to a questionable approximation 
of conservation properties, especially the incompressibility relation (10). This is all the more troublesome as the velocity field $\boldsymbol{u}$ is likely to transport the stress tensor $\tau$ in relation (8).

We present in this section an original mixed finite element method for the approximation of the viscoelastic fluid flow problems. This approximation is based on a combination of Thomas-Raviart element [14, ch. 3] for velocity-pressure fields, piecewise constant functions for normal stress components, and linear continuous functions for the shear stress components (see Fig. 7). We have shown in Ref. [36] that this method leads to a well-posed problem for $W e=0$.

For $W e \neq 0$, a scalar transport term $(\boldsymbol{u} \cdot \nabla) \tau_{i j}$ appears for each component $\tau_{i j}$ of the extra-stress tensor $\tau$. We use the Lesaint-Raviart scheme [28] for the transport term of the normal stress components $\tau_{11}$ and $\tau_{22}\left(\tau_{r r}, \tau_{z z}\right.$ and $\tau_{\theta \theta}$ in the axisymmetric case). These approximations are generally discontinuous at interfaces between elements. Upwinding techniques are possible in schemes involving right-side or left-side values, according to the direction of the flow. We use the Bab-Tabata scheme [1,34], based on a finite volume element formulation on an approximate Riemann solver, for the shear component $\tau_{12}\left(\tau_{r z}\right.$ in the axisymmetric case). This approximation of $(\boldsymbol{u} \cdot \nabla) \tau$ is used for all three steps of the $\theta$-method.

It should be pointed out that the incompressibility relation is exactly satisfied when using the Thomas-Raviart element, and both Lesaint-Raviart and BabaTabata schemes are TVD (total variation diminishing), so no artificial numerical oscillations can occur. This is an improvement on the previous method [35], where the SUPG method [6] was used for approximation of the transport term of shear stress component. In fact, the SUPG scheme is not TVD [19], and numerical oscillations can occur near singularities, associated with strong effects at high Weissenberg numbers.

Moreover, this finite element method was found to be of optimal cost in terms of the total number of degrees of freedom versus the number of element of mesh: six or seven degrees of freedom per element, for six or seven scalar fields, in plane or axisymmetric geometry, respectively.

Table 1 gives a comparative synthesis of this cost with other authors. The test value $\left(N_{\text {dof }} / 6 N_{\text {elem }}\right)_{x}$ is the asymptotic value of the number of degrees of freedom $N_{\text {dof }}$ versus the number of elements $N_{\text {elem }}$ and the number of scalar fields: (six, for

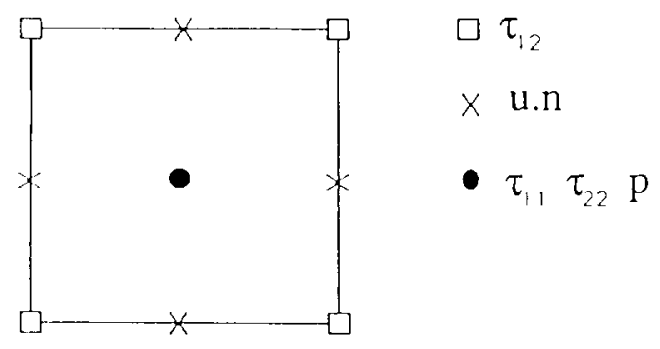

Fig. 7. Incompressible finite element. 
Table 1

Comparative cost of three finite element methods

\begin{tabular}{llll}
\hline$N_{\text {elem }}$ & $N_{\text {dof }}$ & $\left(N_{\text {dof }} / 6 N_{\text {elem }}\right)$, & Ref. \\
\hline 210 & 16719 & $9.5(57 / 6)$ & {$[7]$} \\
326 & 19532 & $9.83(59 / 6)$ & {$[10]$} \\
1144 & 23645 & $3.33(20 / 6)$ & {$[11]$} \\
1196 & 7403 & 1 & This work \\
\hline
\end{tabular}

planar geometry): $\tau_{11}, \tau_{12}, \tau_{22}, u_{1}, u_{2}$, and $p$. We use the Euler relation $N_{\text {edge }}+1=$ $N_{\text {clem }}+N_{\text {node }}$ and suppose that the mesh is regular, made only of quadrangles, and satisfies $N_{\text {node }}=C\left(N_{\text {elem }}\right)$.

Finally, this element can be easily extended to approach three-dimensional problems, always with an optimal cost.

\section{Numerical experiments in an abrupt contraction}

We now describe the application of both the $\theta$-method and the incompressible finite element method to the computation of viscoelastic flows in an axisymmetric contraction. We recall briefly the main features of such flows, and give the conditions of our numerical experiments on the Phan-Thien-Tanner model.

\subsection{General features of the flow in an abrupt contraction}

Such a flow is of greatest interest from both theoretical and practical points of view (e.g. in relation to polymer processing problems) and has been investigated in numerous experimental works. In the entry flow region, before the contraction, the fluid particles are accelerated close to the central part of the duct, while vortices appear close to the edges (see Fig. 8).

This flow can be considered as a complex one, because it is roughly a shear flow close to the solid wall, and becomes rather elongational at the vicinity of the centre. Experiments related to the axisymmetric contraction have shown increasing recirculating zones when the Weissenberg number $W e$ increases. We refer to the recent paper of Binding [3] for a review and an analysis of such intriguing flow behaviours.

\subsection{Numerical and geometrical conditions}

In our numerical experiments, the abrupt contraction was considered to be axisymmetrical $\left(x_{1}=z, x_{2}=r, x_{3}=\theta\right)$, involving an axis of symmetry $r=0$. We may consider a half-flow domain $\Omega$, as shown in Fig. 9.

We have chosen the upstream radius $R=4$, and the downstream radius $r=1$. This corresponds to the classical four-to-one contraction generally investigated in numerical works. Both upstream and downstream lengths are assumed to be 


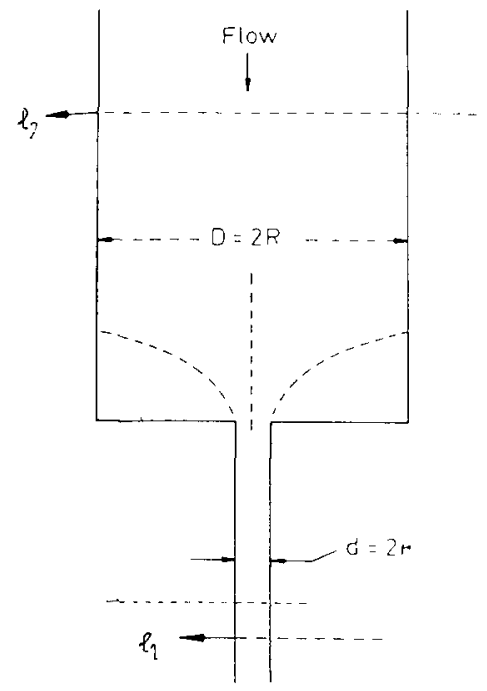

Fig. 8. Description of the $4: 1$ abrupt contraction (from Ref. [3], p. 29).

sufficiently long so that fully developed conditions can exist; we have chosen $l_{1}=64$ and $l_{2}=200$.

\subsection{Choice of material parameters}

The Phan-Thien-Tanner model predicts realistic elongational viscosity, which reaches a maximum (see also Section 3.1). Furthermore, the choice $\varepsilon=0.015$ with an exponential coefficient is consistent with data for polymer melts [26, p. 205]. Moreover, this choice, also used in Ref. [8], is convenient for a cross-checked approach.

The use of the derivative parameter $a$ allows us to control the level of shear thinning (see Section 3.2): $a \neq 0$ and $|a|<1$ lead to non-vanishing second normal stress difference and bounded values of $W e$ at high shear rates; $0.8 \leq a<1$ is consistent with the experimental data. The results of Section 7 use $a=0.8$.

The choice $0<\alpha<\alpha_{c}(\varepsilon)$ guarantees a strictly monotonically increasing shear stress, and the existence of one unique regular solution of the Poiseuille flow

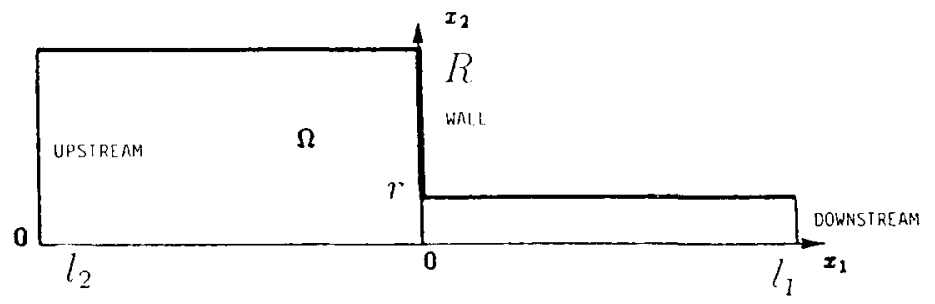

Fig. 9. Specification of the domain of computation. 

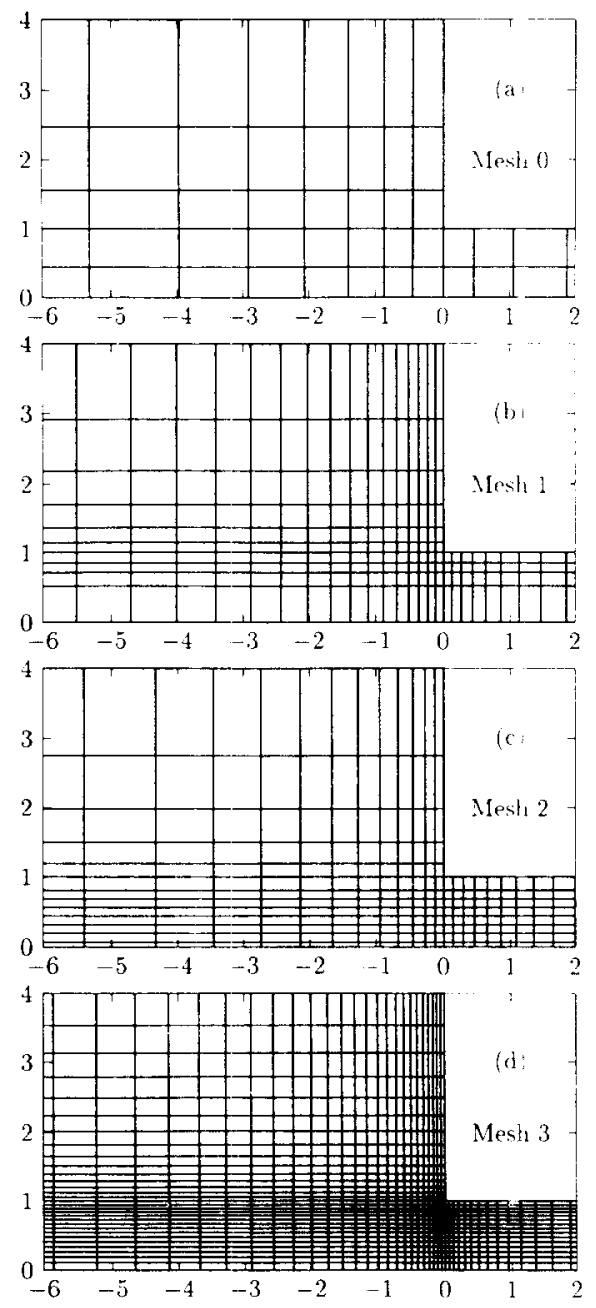

Fig. 10. The finite element mesh family.

problem (see Section 3.3). The choice $x=7 / 8$, near the critical value, satisfies those properties, and allows important viscoelastic effects.

The Weissenberg number is defined by (19), considering the downstream Poiseuille flow. Here, the Reynolds number has no influence on the stationary solution, since only slow flows are investigated: the inertia term $(\boldsymbol{u} \cdot \boldsymbol{\nabla}) \boldsymbol{u}$ in (9) is neglected.

\subsection{Mesh family}

Starting from a rough mesh $\mathscr{T}^{(0)}$, we build a family $\left(\mathscr{T}^{(i)}\right)_{i \geq 0}$ by using a refining procedure of $\mathscr{T}^{(0)}$ near the re-entrant corner (see Fig. 10). 
On $\mathscr{T}^{(i)}$, the length of the element close to the re-entrant corner is given by

$$
h_{i} \approx \frac{r}{3 \times 2^{i}} \text {. }
$$

The sizes of the elements are given by a geometric progression of constant factor, starting from the re-entrant corner.

\section{Phan-Thien-Tanner fluid in an axisymmetric contraction}

We present the computation of the flow through a four-to-one axisymmetric contraction of a Phan-Thien-Tanner fluid with a constitutive equation given by (4). Section 6.3 develops the main motivation for this choice.

\subsection{Convergence tests}

The velocity and stress profiles are given by reference to the average velocity $\bar{U}$ at the downstream section. The mesh convergence properties can be observed by drawing velocity profiles along the axis of symmetry for the meshes 1, 2 and 3 (Fig. 11). We notice an important overshoot near the entry section. We also notice that the mesh convergence of the solution is remarkable for fine meshes 2 and 3, while the rough mesh 1 gives only qualitative results; the convergence velocity of the mesh family is considerable.

Fig. 12 presents the first normal component of the extra-stress tensor along the line $r=1$ close to the re-entrant corner, for the three meshes 1,2 and 3 . In the vicinity of the re-entrant corner, stresses become singular, and the theoretical value is infinite at the corner. As expected, the peak of the stress approximation becomes sharper with the mesh refinement near the singularity. Nevertheless, we can check that convergence occurs outside the vicinity of the re-entrant corner.

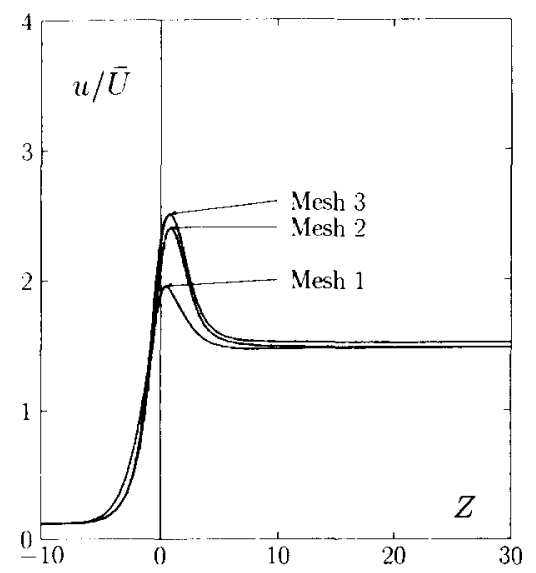

Fig. 11. Mesh convergence of the velocity profile along the axis of symmetry at $i \overline{\gamma_{w}}=9.30:$ meshes 1,2 and 3 . 


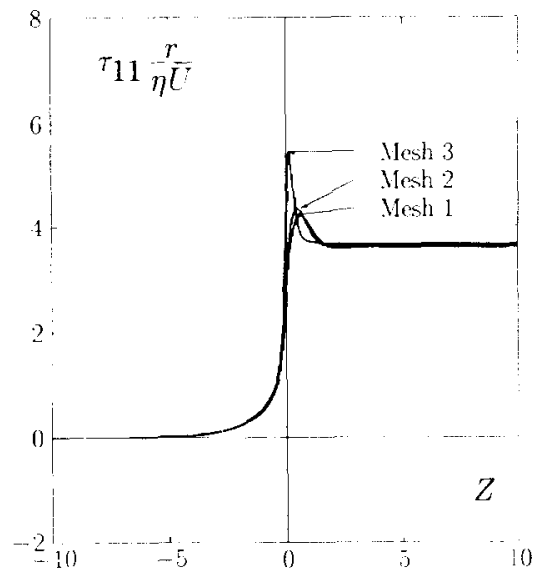

Fig. 12. Mesh convergence of the first normal stress component along the line $r=1$ close to the re-entrant corner at $\hat{i} \dot{i}_{w}=4.50$ : meshes 1,2 and 3 .

\subsection{Velocity and stress profiles}

Let us observe in Fig. 13 the velocity profile along the axis of symmetry as a function of $2 \overline{\gamma_{w}}$. At $W e=0$, a tiny overshoot of the velocity profile theoretically exists, but is not perceptible in Fig. 13(a). From small values of $i \overline{\bar{\gamma}_{\mathrm{w}}}>0$ (Fig. 13b), an overshoot of the velocity clearly appears near the entry section. This overshoot increases with $\overline{i \hat{\gamma}_{\mathrm{w}}}$ (Fig. 13c).

Fig. 14 presents the first normal component profile of the extra-stress tensor along the line $r=1$ close to the re-entrant corner, as a function of $W e$. At $W_{c}=0$ (Fig. 14a), the peak of the Newtonian extra-stress takes negative values, but from small values of $W e>0$, the extra-stress profile changes type, and the peak becomes positive (Figs. 14b,c). As expected, the relaxation process of the stresses along the

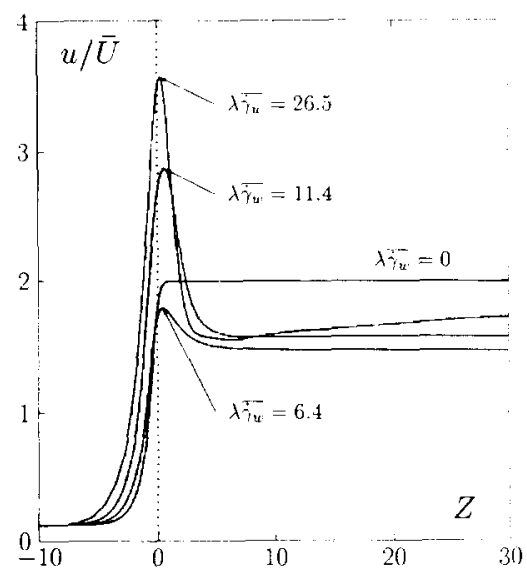

Fig. 13. Velocity profile along the axis of symmetry. as a function of $\lambda \overline{\gamma_{w}}$ (mesh 3 ). 


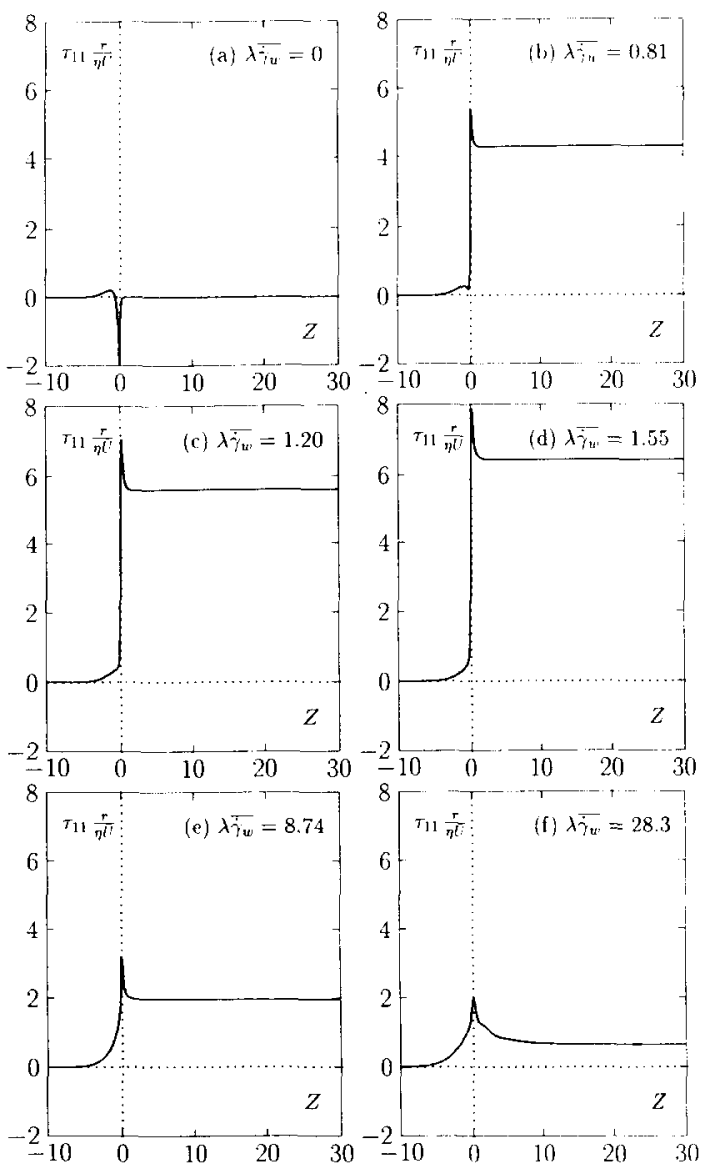

Fig. 14. First normal stress component along the line $r=1$ close to the re-entrant corner, as a function of We (mesh 3).

wall after the re-entrant corner becomes slower as We increases; the downstream length must be sufficiently long for fully developed stress conditions to exist.

Note that the dimensionless first normal component of the extra-stress tensor $\tau_{11} \cdot r / \eta \bar{U}$ at upstream grows to a maximum value (Fig. 14d), and starts to decrease for higher values of $i \dot{\gamma}_{w}$ (Fig. 14e). For high values of the flow rate (see Fig. 14f) this quantity tends to zero, as predicted by relation (17); the lack of interest of the branch of solutions for large values of $\lambda \overline{\dot{\gamma}_{w}}$ could define a limit of the Phan-ThienTanner model for high flow rates.

\subsection{Vortex development and intensity}

Fig. 15 shows the vortex development as a function of $W e$ and $\lambda \overline{\dot{\gamma}_{w}}$. For $W e=0$ (Newtonian fluid), we observe a small vortex close to the salient corner of the contraction. The change in concavity can be observed between Figs. 15(b) and (c). We notice in Figs. 15(c) and (d) the growth of the vortex size. The development of 
the vortex reaches a maximum (nearly Fig. 15d) and is followed by a slow decrease for high values of the flow rate.

Figs. 16(a) and (b) present the vortex instensity as functions of $\overline{i \dot{\gamma}_{u}}$ and $W e$. respectively. The vortex intensity is defined by the ratio of the recirculating flow rate in the vortex to the flow rate through the contraction. Vortex intensity grows. reaching a maximum value for $\bar{\lambda} \overline{\dot{\gamma}_{\mathrm{w}}} \approx 13.8(W e=6.60)$, and then slowly decreases to the asymptotic Newtonian value. When $\overline{i \bar{i}_{w}} \approx 13.8$, the vortex intensity reaches nearly $10 \%$ of the main flow rate; the corresponding Newtonian value is nearly $0.2 \%$. Note that in Fig. 15(b) the variations of We fits the variations of the vortex

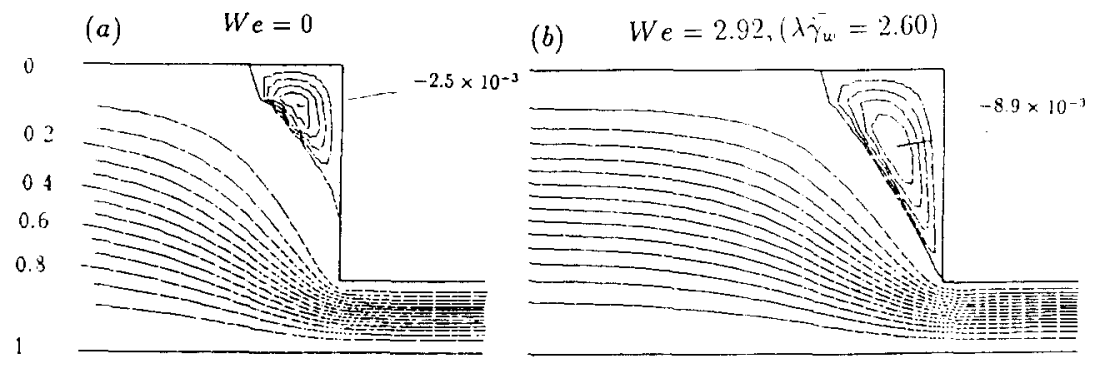

(c) $W_{\epsilon}=5.84,\left(\lambda \dot{\gamma_{w}}=7.60\right)$

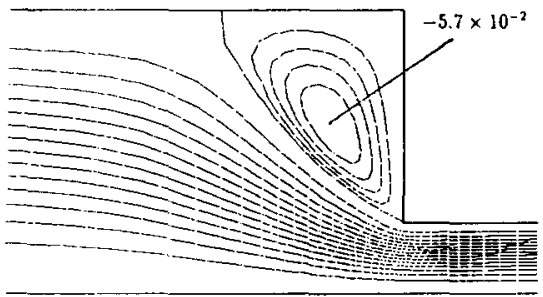

(d) $W e=6.55,\left(\lambda \dot{\gamma}_{w}=11.4\right)$

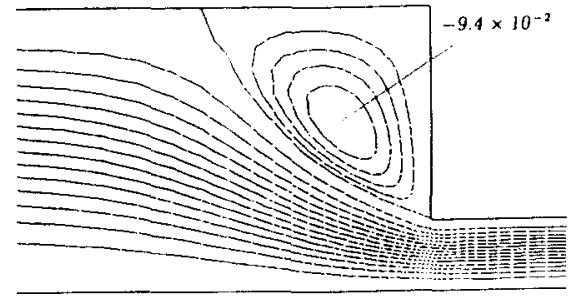

(e) $W e=5.30,\left(\lambda \dot{\gamma_{w}}=29.2\right)$

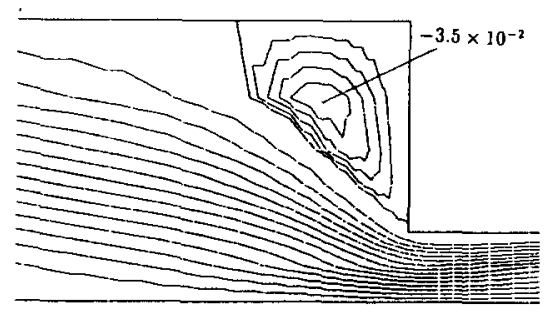

Fig. 15. Vortex developments as a function of We. 

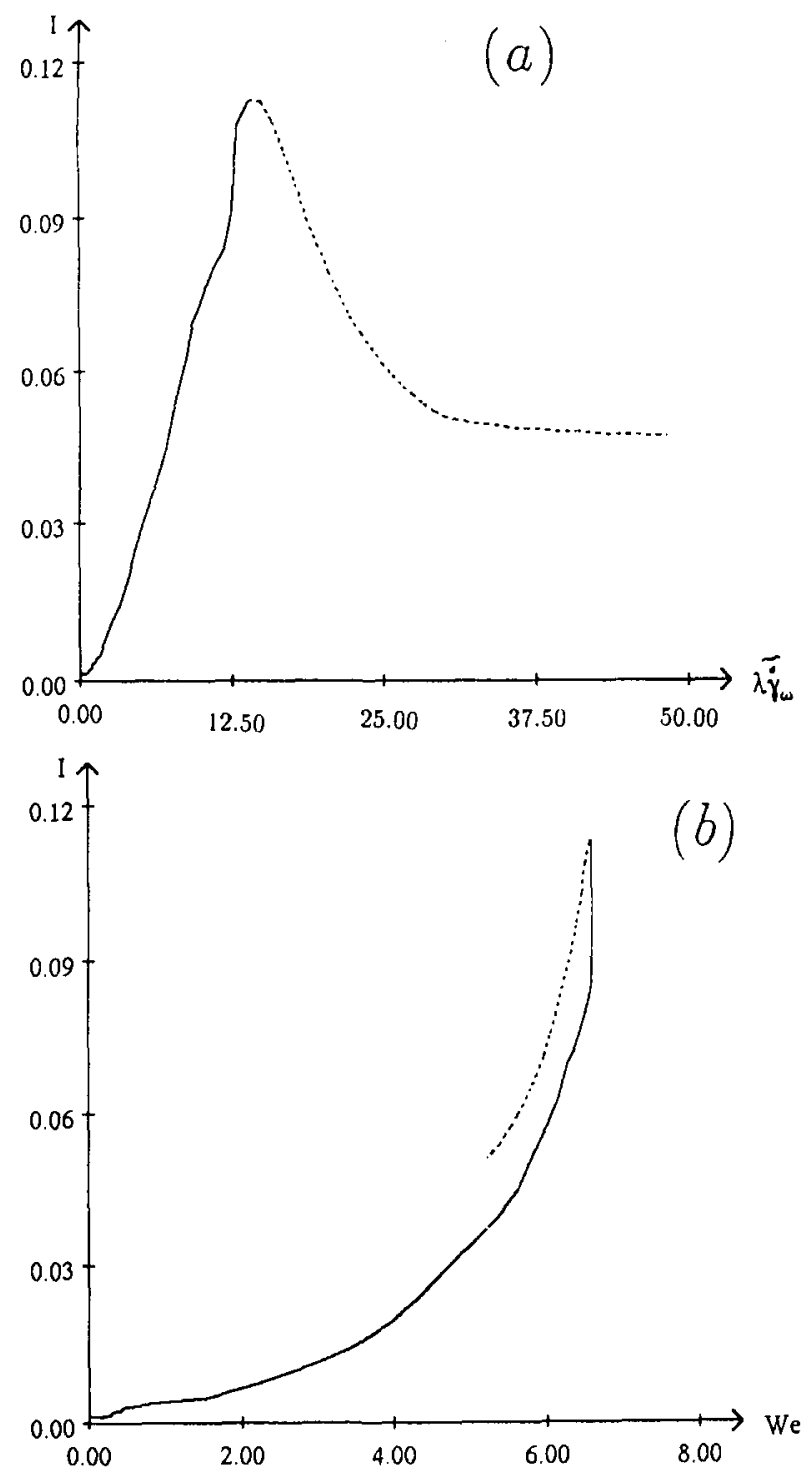

Fig. 16. Vortex intensity as a function of $\lambda \overline{\gamma_{w}}$ (a) and $W e(b)$.

development (see also Fig. 6). This leads to argue that both the Weissenberg number (defined by relation 19) and the vortex activity of converging flows are related to the elongational properties of Phan-Thien-Tanner fluids.

These numerical results have been found to be qualitatively and quantitatively consistent with those of ref. [8]. Note that in Ref. [8] the authors used $W e^{*} \stackrel{\text { def }}{=} \lambda \bar{U} / r$ as a definition of the Weissenberg number, where $\bar{U}$ is the average velocity at the downstream section, and $r$ is the radius of the downstream section $\left(W e^{*}=\lambda \overline{\dot{\gamma}_{w}} / 4\right.$ for an axisymmetric contraction, and $W e^{*}=\lambda \overline{\dot{\gamma}_{w}} / 3$ for a plane contraction). As a 
Table 2

Comparative efficiency of three decoupled algorithms

\begin{tabular}{lc}
\hline Algorithm & No. of iterations \\
\hline Picard method & 2100 \\
GMRES method & $210(7 \times 30)$ \\
0 -method & $42(21 \times 2)$ \\
\hline
\end{tabular}

consequence, the Weissenberg number, as defined in Ref. [8], is a linear function of the flow rate.

\section{Conclusions}

The efficiency of our time-dependent decoupled approach ( $\theta$-method) is confirmed by the numerical results of this paper. The $\theta$-method is used here as a fast algorithm for reaching stationary solutions.

However, in Ref. [8], Debbaut et al. used a coupled Newtonian method. This approach, combined with a sub-element approximation of stress components, requires long computation times on a supercomputer. Following Fortin et al. [10], we point out that decoupled methods seem to be the only realistic alternative for three-dimensional flow problems.

Combining the $\theta$-method with the finite element approximation presented in Section 5, we obtain a fast solver for viscoelastic fluid flow problems. This method is shown to be much more efficient than the Picard or the GMRES method [10]. Using the plane four-to-one abrupt contraction, the Oldroyd-B model and starting with stationary solution at $\lambda=2.5$ as in Ref. [10], the $\theta$-method converges in just 21 time steps to the stationary solution at $\lambda=2.75$. After 21 time steps the $L^{2}$ norm of the residual term of the approximate stationary system takes the value of $9.1 \times 10^{-4}$. One time step is roughly equivalent, from a computational point of view, to two Picard iterations. Table 2 gives comparative test results of these three decoupled algorithms. We can conclude that the $\theta$-method is five times faster than GMRES method, and 50 times faster than the Picard method.

The good quality of numerical solutions given by TVD schemes for the stress transport term is exhibited: in particular, we observe no numerical oscillations near the re-entrant corner at high values of the flow rate, which is not the case in previous works $[7,11,35]$.

Finally, the main flow features of the Phan-Thien-Tanner fluid are presented. This model allows realistic predictions of elongational properties and shear thinning. We have introduced a new definition of the Weissenberg number in converging flows. This definition takes into account the elongational properties of the flow. Moreover, we have shown that this dimensionless number fits the vortex activity in an axisymmetric abrupt contraction. 


\section{Acknowledgements}

J.M. Piau helped me greatly in preparing and reviewing this paper. The author would like to thank C. Guillopé and J.C. Saut for fruitful discussions, and H. Garavel and R. Michel for their careful reading of the paper.

\section{References}

[1] K. Baba and M. Tabata, On a conservative upwind finite element scheme for convection diffusion equations. RAIRO Numer. Anal., 15 (1981) 3-25.

[2] J. Baranger and D. Sandri, A formulation of the Stokes problem and the linear elasticity equation suggested by the Oldroyd model for viscoelastic flows, Math. Modelling Num. Anal., 26 (1992) $331-345$.

[3] D.M. Binding, Further considerations of the axisymmetric contraction, J. Non-Newtonian Fluid Mech., 41 (1991) 27-42.

[4] D.V. Boger, M.J. Crochet and R.A. Keiller, On viscoelastic flows though abrupt contractions, J. Non-Newtonian Fluid Mech., 44 (1992) 257-279.

[5] F. Brezzi, On the existence, uniqueness and approximation of saddle point problems arising from Lagrangian multiplier, RAIRO, série Analyse Numérique, R2 (1974) 129-151.

[6] A.N. Brooks and T.R.J. Hughes, Streamline-upwind/Petrov-Galerkin formulation for convection dominated flow with particular emphasis on the incompressible Navier-Stokes equations, Comp. Meth. Appl. Mech. Eng., 32 (1982) 199-259.

[7] M.J. Crochet and J.M. Marchal, A new mixed finite element for calculating viscoelastic fluid flows, J. Non-Newtonian Fluid Mech., 26 (1987) 77-114.

[8] B. Debbaut, J.M. Marchal and M.J. Crochet, Numerical simulation of highly viscoelastic flows though an abrupt contraction, J. Non-Newtonian Fluid Mech., 29 (1988) 119-146.

[9] J.A. Deiber and W.R. Schowalter, On the comparison of simple non-monotonic constitutive equations with data showing slip of well-characterized polybutadienes, J. Non-Newtonian Fluid Mech., 40 (1991) 141-150.

[10] A. Fortin and A. Zine, An improved GMRES method for solving viscoelastic fluid flow problems, J. Non-Newtonian Fluid Mech., 42 (1992) 1-18.

[11] M. Fortin and A. Fortin, A new approach for the FEM simulation of viscoelastic flows, J. Non-Newtonian Fluid Mech., 32 (1989) 295-310.

[12] M. Fortin and R. Glowinski, Lagrangian Augmented Methods, North-Holland, Amsterdam, 1981.

[13] M. Fortin and R. Pierre, On the convergence of a mixed method of Crochet and Marchal for viscoelastic flows, Comp. Meth. Appl. Mech. Eng., 73 (1989) 341.

[14] V. Girault and P.A. Raviart, Finite Element Approximation of the Navier-Stokes Equations, Lecture Notes in Mathematics, 1979.

[15] R. Glowinski and J. Périaux, Numerical methods for nonlinear problems in fluid dynamics, in: Proc. Int. Seminar on Scientific Super-Computers, Feb. 1987.

[16] R. Glowinski and O. Pironeau, Ann. Rev. Fluid Mech., 24 (1992) 167-204.

[17] R. Glowinski and P. Le Tallec, Augmented Lagrangian and Operator Splitting Method in Non-Linear Mechanics, SIAM Studies in Applied Methematics, 1989.

[18] G.H. Golub and A. Meurant, Résolution numérique des grandes systèmes linéaires, CEA-EDFINRIA, 1983.

[19] J.B. Goodman and R.L. Leveque, A geometric approach to high resolution TVD schemes, SIAM J. Numer. Anal., 25 (1988).

[20] C. Guillopé and J.C. Saut, Résultat d'existence pour les fluides viscoélastiques à loi de comportement de type différentiel, C. R. Acad. Sci. Paris, I, 305 (1987) 489-492.

[21] C. Guillopé and J.C. Saut, Global existence and one-dimensional non-linear stability of shearing motions of viscoelastic fluids of Oldroyd type. Math. Modelling Num. Anal., 24 (1990) 369-401. 
[22] D.D. Joseph, M. Renardy and J.C. Saut, Hyperbolicity and change of type in the flow of viscoelastic fluids, Arch. Ration. Mech. Anal., (1985) 213 251.

[23] R. Keunings, Fundamentals of Computer Modeling for Polymer Processing, ed. C.L. Tucker III. Carl Hanser Verlag. 1988.

[24] N. El Kissi and J.M. Piau, Ecoulement de fluides polymères enchevêtrés dans un capillaire. modélisation du glissement macroscopique à la paroi. C. R. Acad. Sci. Paris. 309 (1989) 79.

[25] N. El Kissi and J.M. Piau, The different capillary flow regimes of entangled polydimethylsiloxane polymers: macroscopic slip at the wall, hysteresis and cork flow. J. Non-Newtonian Fluid Mech., 37 (1990) $55-94$.

[26] R.G. Larson. Constitutive Equations for Polymer Melts and Solutions. Butterworths Ser. Chem. Eng. H. Brenner, 1988.

[27] A.I. Leonov, Analysis of simple constitutive equations for viscoelastic liquids, J. Non-Newtonian Fluid Mech., 42 (1992) 323-350.

[28] P. Lesaint and P.A. Raviart. On Finite Element Methods for Solving the Neutron Transport Equation, Academic Press, 1974.

[29] D.S. Malkus, M.G. Hansen, G.R. Ierley and R.A. Worthing. Spurt phenomena of the Johnson Segalman fluid and related models, J. Non-Newtonian Fluid Mech., 29 (1988) 303- 335.

[30] J.G. Oldroyd, On the formulation of rheological equations of states, Proc. R. Soc. London. A. 200 (1950) $523-541$.

[31] D.W. Peaceman and H.H. Rachford. The numerical solution of parabolic and elliptic differential equations, J. Soc. Ind. Appl. Math., 3 (1955) $28-41$.

[32] M. Renardy, Existence of slow flows of viscoelastic fluids with differential constitutive equations. $Z$. Angew. Math. Mech., 65 (1985) 449-451.

[33] M. Renardy, Recent advances in the mathematical theory of steady flows of viscoelastic fluids, I. Non-Newtonian Fluid Mech., 29 (1988) 11-24.

[34] P. Rostand and B. Stoufflet, Finite volume Galerkin methods for viscous gas dynamics. R R INRIA (1988) 863.

[35] P. Saramito, Simulation numérique d'écoulements de fluides viscoélastiques par éléments finis incompressibles et une méthode de directions alternées; applications. Thèse de l'Institut National Polytechnique de Grenoble, 1990.

[36] P. Saramito, Numerical simulation of viscoelastic fluid flows using incompressible finite element method and a $\theta$-method. Math. Modelling Num. Anal., 35 (1994) I 35.

[37] P. Saramito. Operator splitting for viscoelastic fluid with a differential constitutive law, C. R. Acad. Sci. Paris, II (1994) 267-270.

[38] N. Phan-Thien and R.I. Tanner, A new constitutive equation derived from network theory, J. Non-Newtonian Fluid Mech., 2 (1977) 353-365.

[39] G.V. Vinogradov, V.P. Protasov and V.E. Dreval, The rheological behavior of flexible-chain polymers in the region of high shear rates and stresses, the critical process of spurting. and supercritical conditions of their movement at $T>T_{\underline{\underline{g}}}$. Rheol. Acta, 23 (1984) $46-61$. 